\title{
Generalized van der Waals theory for phase behavior of two-dimensional nematic liquid crystals: Phase ordering and the equation of state
}

\author{
María Virginia Zonta and Ezequiel Rodolfo Soule* \\ Institute of Materials Science and Technology (INTEMA), University of Mar del Plata \\ and National Research Council (CONICET), \\ J. B. Justo 4302, 7600 Mar del Plata, Argentina
}

(Received 22 October 2019; published 26 December 2019)

\begin{abstract}
Liquid crystalline ordering of anisotropic particles in two dimensions is important in many physical and biological systems and their phase behavior is still a topic of interest. A generalized van der Waals theory is formulated, accounting for repulsive excluded volume and attractive van der Waals and Maier-Saupe interactions, for rectangles confined to two dimensions. The phase ordering transitions and equation of state are analyzed as a function of the model parameters (aspect ratio $L / B$ and isotropic and anisotropic interaction parameters $\chi$ and $v$ ). Different phase transitions are observed: continuous isotropic-nematic (high $L / B$ and $v$ ), first-order isotropic-nematic (intermediate $L / B$ and small $v$ ), and continuous isotropic-tetratic (small $L / B$ and $v$ ) followed by a continuous tetratic-nematic transition at higher densities. Increasing $L / B$ decreases the pressure, and this effect is more pronounced in the nematic than in the isotropic phase. Increasing both interaction parameters decreases pressure and can lead to phase separation.
\end{abstract}

DOI: 10.1103/PhysRevE.100.062703

\section{INTRODUCTION}

The study of ordering transitions in two dimensions is relevant for many physical systems. Some two-dimensional (2D) or quasi-2D systems are molecular layers, membranes, molecules (or particles) adsorbed in a surface, or even strongly confined thin films. In-plane two-dimensional nematic order can be found whenever elongated (e.g., fiberlike or rodlike) particles are confined to a surface, membrane, or layer, with the long axis parallel to the plane of that surface: that is the case of macromolecules adsorbed to a surface or interface [1-4], particles confined in layers [5], or films of anisotropic bacteria [6], to mention a few examples.

For three-dimensional (3D) nematic ordering, there are two theoretical approaches than can be considered to be seminal works: Onsager theory [7], which is based purely in excluded volume interactions, and Maier-Saupe theory [8,9], which only accounts for attractive quadrupolar interactions. Onsager theory was formulated within a second virial approximation, so it is valid for particles with high aspect ratio (isotropicnematic transition at low concentrations). There are, however, other more accurate approaches to excluded volume interactions, like Parsons-Lee approximation [10,11], that overcome this limitation. These theories show that nematic ordering can be induced only by increasing concentration. On the other hand, the Maier-Saupe approach completely neglects excluded volume effects so it is (in principle) valid only for very short rods (with anisotropic attractive interactions), and it is used to describe thermal transitions. Generalized van der Waals theories, which combine excluded volume and attrac-

*ersoule@fi.mdp.edu.ar tive interactions, have been developed as well [12-15] and used to describe phase transitions as a function of temperature and density.

Compared to 3D systems, in-plane liquid crystals are simpler in many aspects due to reduced dimensionality, but they show a complexity not present in higher dimensions: strictly speaking, true long-range order does not exist in two dimensions. Nevertheless, in some cases the difference between quasi-long-range order (with an algebraic decay in correlations) and true long-range order is not relevant, and mean-field theories, which assume true long-range order, provide a satisfactory description of some aspects of the behavior of 2D systems [16,17]. In addition, many systems with 2D order are actually "quasi-2D systems" due to interactions in a third dimension; that is the case of systems that are in equilibrium with a solution, the case of strong but not complete confinement to two dimensions where fluctuations in the third dimension are possible, or interacting multilayers. Considering this, mean-field theories for 2D order have been used to study many physical systems like fiberlike or rodlike particles or molecules adsorbed to surfaces [18-21], confined systems [5,22], lipid layers, and biological membranes [23-25], etc.

Monte Carlo simulations by Frenkel and coworkers $[17,26,27]$ of hard-core $2 \mathrm{D}$ systems showed that a quasinematic phase with algebraic order exists for large aspect ratios, while for short particles (with aspect ratio $L / B<5$ ) the ordered phase seems to be crystalline or smectic. In the former case, the order-disorder transition is continuous, while in the latter it is first order (as opposed to 3D systems where the transition is always first order). Different theoretical mean-field approaches, based in density functional theory [28], second virial (Onsager) approximation [29], and scaled particle theory [16], found also a continuous transition. It 
was shown that these mean-field approaches reproduce the equation of state and the qualitative behavior of the order parameter fairly well, despite the fact that they assume longrange order. As expected, the order-disorder transition moves to higher densities as $L / B$ decreases. Schlacken and Mogel found that, for very short rods, the transition from isotropic to nematic (twofold symmetry) phase is replaced by an isotropic to tetratic (fourfold symmetry) transition [16]. This is to be expected, considering that for $L / B=1$ rectangles become squares. For intermediate $L / B$, the isotropic to nematic transition becomes first order.

In a different approach, particles with attractive directional interactions but no excluded volume have been considered. Theory and simulation using a generalized $X Y$ model found that when interactions are "sharp and narrow" (particles interact only when they are highly aligned) the transition is first order, while for interactions that depend softly on orientation (like the standard $X Y$ model or the Maier-Saupe case) the transition is continuous [30,31]. Again, nematic order is found to be algebraic. Geng and Selinger [32] specifically considered the tetratic phase, by using an interaction of the MaierSaupe type, considering terms representing both twofold and fourfold anisotropic interactions. They calculated phase diagrams that showed a low-temperature nematic phase, a hightemperature isotropic phase, and for a symmetry breaking parameter (the ratio between fourfold and twofold interactions) higher than a critical value an intermediate tetratic phase. For values of the symmetry breaking parameter close to this critical value, the transition is first order, while it is continuous for large or small enough values of this parameter.

Both types of interactions (repulsive and attractive) were considered by Heinemann et al. [33], who performed molecular dynamics simulations for ellipses with linear quadrupolar interactions confined to a plane. They found that, for relatively small aspect ratios, a plethora of ground state crystalline structures can exist, and they persist to finite temperatures. They did not, however, focus specifically on liquid-crystalline phases.

In this paper, a generalized van der Waals theory for liquid crystalline order in a system of elongated particles confined to two dimensions is presented. Scaled particle theory is used for excluded volume repulsive interactions, while attractive interactions are described by a Maier-Saupe quadrupolar term and an isotropic van der Waals interaction term. The orderdisorder transition and equation of state are analyzed in the whole range of geometrical and interaction parameters. This combines many of the aspects considered in previous works in a single generalized and relatively simple theoretical framework, allowing one to assess the relative importance of excluded volume and attractive interactions.

\section{MODEL AND METHODS}

\section{A. Free energy}

A system of $N$ moles of hard rectangles in a surface of area $A$ is considered. The free energy $F$ is written in a van der Waals approach [12-15] by combining scaled particle [16] theory, which accounts for hard-core interactions, with a Maier-Saupe (anisotropic) and van der Waals type (isotropic) of attractive interactions:

$$
\frac{F}{N R T}=\ln \phi-\ln (1-\phi)+\sigma+\frac{\phi}{1-\phi} \rho-\phi a_{r}\left(\chi^{\prime}+v^{\prime} S_{2}^{2}\right),
$$

where $\phi$ is the surface density, $a_{r}=B L$ is the area of the rectangle, and $B$ and $L$ are the width and length of the rectangle. The first four terms (scaled particle theory) are purely entropic, the first two correspond to translational entropy, while the third and fourth represent the orientational and excluded volume free energy and are given by

$$
\begin{gathered}
\sigma=\int_{0}^{\pi} f(\theta) \ln \pi f(\theta) d \theta \\
\rho=\int_{0}^{\pi} d \theta \int_{0}^{\pi} f(\theta) f\left(\theta^{\prime}\right) K\left(\theta-\theta^{\prime}\right) d \theta^{\prime}
\end{gathered}
$$

where $f$ is the orientational distribution function. The kernel $K$ is related to the excluded area between two particles with a relative orientation $\gamma=\theta^{\prime}-\theta^{\prime}$; for hard rectangles it is given by [16]:

$$
K(\gamma)=|\cos (\gamma)|+\frac{B^{2}+L^{2}}{2 B L}|\sin (\gamma)|
$$

The last term in Eq. (1) represents the attractive interactions, with two interaction parameters: $\chi^{\prime}$ (isotropic) and $v^{\prime}$ (nematic). Finally, the orientational order parameters are defined as

$$
S_{i}=\int_{0}^{\pi} \cos (i \theta) f(\theta) d \theta
$$

The lowest-degree nonzero order parameter defines the type of phase: in the isotropic phase, where $f(\theta)=1 / \pi, S_{i}=$ 0 for every $i>0$, which also leads to $\sigma=0$ and $\rho=2 / \pi$ $[1+1 / 2(L / B+B / L)]$. The nematic phase is characterized by $S_{2}>0$ (and in general every $S_{i}>0$ ), while in the tetratic phase $S_{2}=0$ and $S_{4}>0$ (and in general $S_{i}>0$ for $i>4$ ). Note that in the case under study, where orientations $\theta$ and $-\theta$ are indistinguishable, odd-degree order parameters $\left(S_{1}, S_{3}\right.$, etc.) are always zero.

This theory, as it considers both hard-core entropic and energetic interactions, can account for concentration-induced (lyotropic) as well as temperature-induced (thermotropic) phase transitions [note that in Eq. (1) the interaction parameters, as defined, should be inversely proportional to temperature]. As positional order is not accounted for in this theory, the purely orientationally ordered phases analyzed in this paper may be metastable with respect to crystalline or smectic order for small $L / B$ or large interactions, where positionally ordered phases were observed in simulations $[17,26,33]$.

\section{B. Phase ordering}

Minimization of the free energy with respect to the orientational distribution function $f$ leads to

$$
\begin{aligned}
& \ln f(\theta)-v^{\prime} B L \phi S \cos (2 \theta) \\
& \quad+2 \frac{\phi}{1-\phi} \int_{0}^{\pi} f\left(\theta^{\prime}\right) K\left(\theta-\theta^{\prime}\right) d \theta^{\prime}=\lambda,
\end{aligned}
$$

where $\lambda$ is a Lagrange multiplier to enforce normalization of the distribution function. 
Equation (6) is solved by expanding the kernel and the distribution function in terms of basis functions:

$$
\begin{aligned}
|\sin (\gamma)| & =\frac{2}{\pi}-\frac{4}{\pi} \sum_{1}^{\infty} \frac{1}{\left(4 n^{2}-1\right)} \cos (2 n \gamma), \\
|\cos (\gamma)| & =\frac{2}{\pi}-\frac{4}{\pi} \sum_{1}^{\infty} \frac{(-1)^{n}}{4 n^{2}-1} \cos (2 n \gamma), \\
f(\theta) & =\frac{\exp \left[\sum_{1}^{\infty} \alpha_{n} \cos (2 n \theta)\right]}{Z} \\
Z & =\int_{0}^{\pi} \exp \left[\sum_{1}^{\infty} \alpha_{n} \cos (2 n \theta)\right] d \theta .
\end{aligned}
$$

Considering the trigonometric identity for the cosine of the difference between two angles,

$$
\cos (\alpha-\beta)=\cos (\alpha) \cos (\beta)+\sin (\alpha) \sin (\beta),
$$

Eq. (6) reduces to

$$
\begin{aligned}
\sum_{1}^{\infty} \alpha_{n} \cos (2 n \theta)-\ln Z \\
=\lambda+v^{\prime} B L \phi S_{2} \cos (2 \theta)-\frac{4}{\pi} \frac{\phi}{1-\phi} \int_{0}^{\pi} f\left(\theta^{\prime}\right) \\
\times\left[1+\frac{\left(B^{2}+L^{2}\right)}{2 B L}-2 \sum_{1}^{\infty}\left(\frac{(-1)^{n}+\frac{\left(B^{2}+L^{2}\right)}{2 B L}}{\left(4 n^{2}-1\right)}\right)\right. \\
\left.\times\left[\cos (2 n \theta) \cos \left(2 n \theta^{\prime}\right)+\sin (2 n \theta) \sin \left(2 n \theta^{\prime}\right)\right]\right] d \theta^{\prime} .
\end{aligned}
$$

The last step is to perform the inner product between Eq. (8) and the basis functions; taking into account that

$$
\begin{aligned}
\int_{0}^{\pi} \cos (2 n \theta) \cos (2 w \theta) d \theta & =\frac{1}{2} \pi \delta_{n w}, \\
\int_{0}^{\pi} \cos (2 n \theta) \sin (2 w \theta) d \theta & =0 \\
\int_{0}^{\pi} \cos (2 w \theta) d \theta & =0
\end{aligned}
$$

the self-consistency equation for the coefficients of the expansion of $f$ is obtained:

$$
\begin{aligned}
\alpha_{2 n}= & \delta_{n 2} v^{\prime} B L \phi S_{2}+\frac{\phi}{1-\phi} \frac{8}{\pi\left(4 n^{2}-1\right)} \int_{0}^{\pi} f\left(\theta^{\prime}\right) \\
& \times\left[\left((-1)^{n}+\frac{B^{2}+L^{2}}{2 B L}\right) \cos \left(2 n \theta^{\prime}\right)\right] d \theta^{\prime},
\end{aligned}
$$

while the odd terms are zero.

The value of the expansion coefficients $\alpha_{n}$ can be calculated for given values of $\phi, L / B$, and $v=v^{\prime} B^{2}$. The infinite expansions are truncated to a number $N$ of terms that allows one to represent the orientational distribution function within a given error tolerance. The value of $N$ was dependent on the values of $\varphi, L / B$, and $v$, and it was large enough such that increasing it did not have a significant effect on the results.

\section{Equation of state}

The pressure $P$ ' can be calculated from the free energy according to

$$
P^{\prime}=\left.\frac{\partial F}{\partial A}\right|_{T, N}
$$

Replacing the expression for the free energy and performing the derivative leads to the following expression for the dimensionless pressure:

$$
P=\frac{B^{2} P^{\prime}}{R T}=\frac{B}{L} \frac{\phi}{(1-\phi)}+\frac{\phi^{2}}{(1-\phi)^{2}} \frac{B}{L} \rho-\phi^{2} B^{2}\left(\chi^{\prime}+v^{\prime} S^{2}\right) .
$$
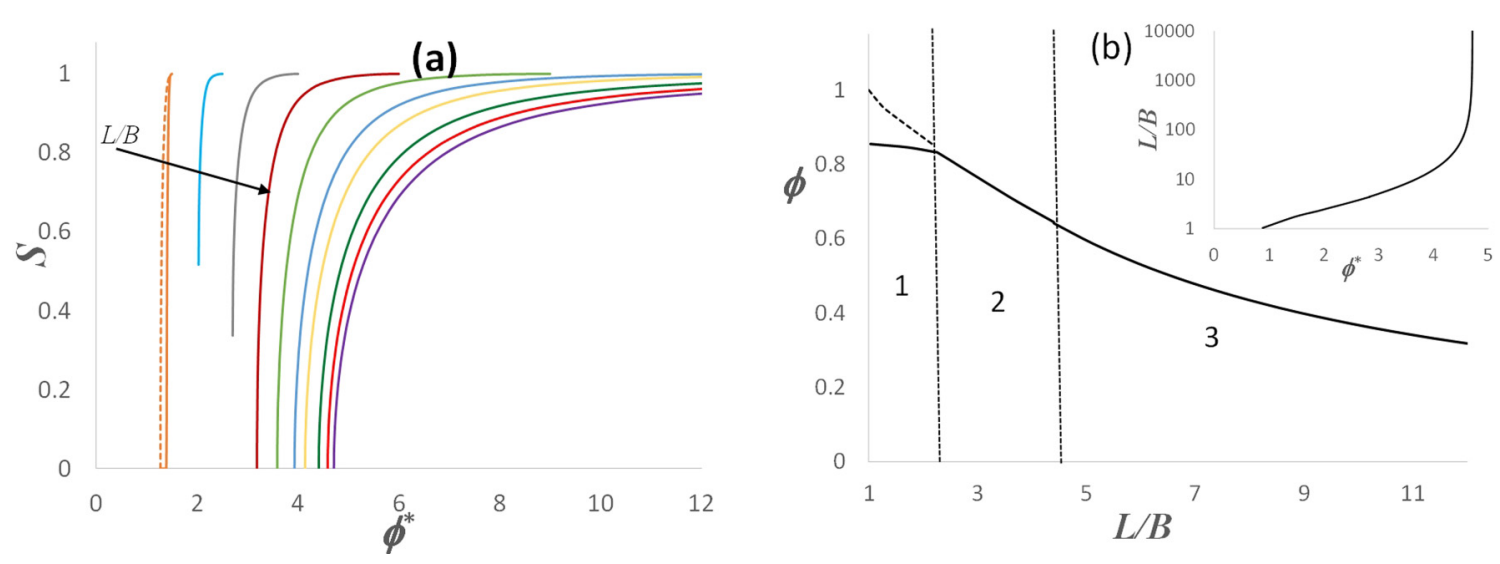

FIG. 1. Order-disorder transition for an athermal system. (a) Order parameter as a function of the scaled area density, for different values of $L / B=1.5,2.5,4,6,9,14,20,40,100$, and 10000 , increasing as indicated by the arrow. The nematic order parameter $S_{2}$ is indicated with a full line and, for $L / B=1.5$, the tetratic order parameter $S_{4}$ is indicated with a dashed line. (b) Area density at the order-disorder transition, $\phi_{\mathrm{IN}}$, for small values of $L / B$. Different ordering transitions (full line) are indicated as follows: in region 1, isotropic-tetratic; in region 2 , discontinuous isotropic-nematic; in region 3, continuous isotropic-nematic. Tetratic-nematic transition is indicated with the dashed line. In the inset, the scaled area density $\phi_{\mathrm{IN}}^{*}$ is shown in the whole range of $L / B$. 


\section{RESULTS AND DISCUSSION}

\section{A. Phase ordering}

First, the equilibrium orientational distribution functions are calculated for different values of the model parameters. As the isotropic interaction, given by $\chi$, does not affect ordering, only the rectangle aspect ratio $L / B$ and the quadrupolar interaction parameter $v$ are considered in this analysis. Some results are presented in terms of the scaled area fraction, $\phi^{*}=\phi L / B$, because in this way the curves converge in the "Onsager" limit (infinite $L / B$ ).

Figure 1 shows the ordering behavior for the athermal case $(v=0)$. In Fig. 1(a) the order parameter as a function of $\phi^{*}$ for different values of $L / B$ is shown, while Fig. 1(b) summarizes the transition points. For very large $L / B$, the transition point and the curves of $S_{2}$ vs $\phi^{*}$ converge to the Onsager limit, as mentioned before, becoming independent of $L / B$. As this parameter decreases, the transition is displaced to smaller $\phi^{*}$ (but larger nonscaled $\phi$, as expected). As it was discussed in the introduction, except for small values of $L / B$ the transition is continuous, that is, $S_{2}$ shows no discontinuity at the transition, although it is noted that the slope of the curve at the transition is infinite, so $S_{2}$ increases quite abruptly in a very small density range. For small values of $L / B$, the behavior of the system is different. In the range $2.18<L / B<$ 4.372, the isotropic-nematic transition becomes first order, and there is a discontinuous jump in $S_{2}$, from zero to a finite value, at the transition. As $L / B$ gets even smaller the shape of the particles approaches a square, which as mentioned in the introduction cannot form a nematic phase. When $L / B<2.18$, an isotropic to tetratic transition is observed, followed by another transition to a nematic phase at larger $\phi$. Both of these transitions are continuous. While, as mentioned before, the nematic phase does not exist for $L / B=1$ (the minimum density for the existence of a nematic phase approaches 1 as $L / B$ approaches 1 ), the tetratic phase does exist when the density is large enough.

Figure 2 shows the behavior when a nonzero quadrupolar interaction is introduced, for a large (a) and a small (b) value of $L / B$. Increasing the quadrupolar interaction favors nematic ordering and thus displaces the curves to smaller densities. Figure 3 shows the transition density $\phi_{\mathrm{IN}}$ as a function of $v$

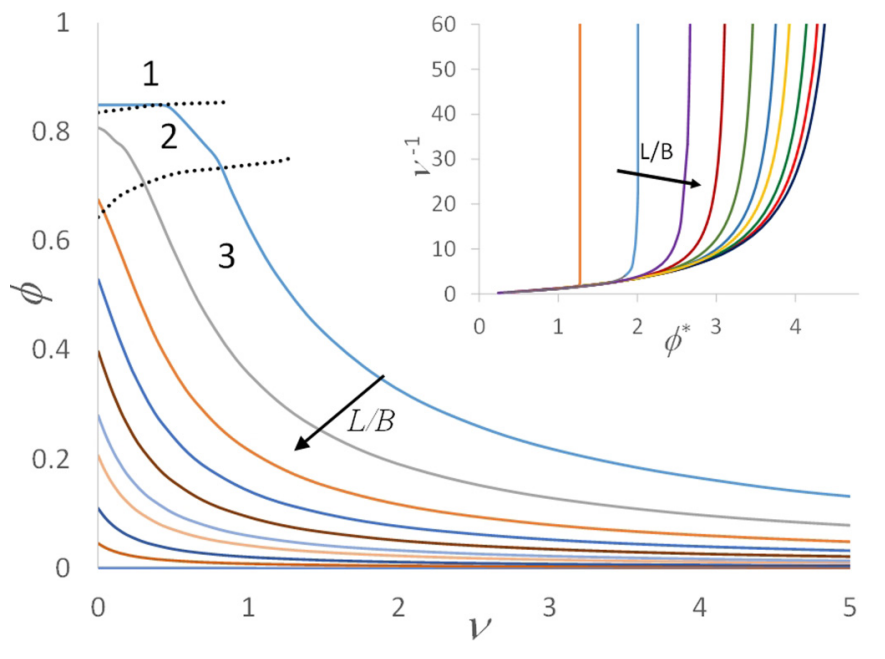

FIG. 3. Density at the order-disorder transition as a function of the quadrupolar order parameter, for the same values of $L / B$ as in Fig. 1. The inset shows the inverse of the quadrupolar interaction parameter vs scaled density at the transition, for the same values of $L / B$ as in Fig. 1. Regions 1, 2, and 3 indicate different phase transitions as in Fig. 1.

where the same is seen. As $v$ goes to infinity, the transition density approaches zero. The effect of increasing $v$ is stronger for large $L / B$, in the sense that a similar relative displacement of the transition density and the $S_{2}$ vs $\phi$ curves requires a smaller $v$ for larger $L / B$, or, in other words, as $v$ decreases the athermal limit is approached faster when $L / B$ is small.

While the nematic phase is favored by the quadrupolar interaction, the tetratic phase is not affected. For small $L / B$ where the tetratic phase is observed in the athermal limit, attractive interactions displace the tetratic-nematic to smaller densities and for large enough $v$ the isotropic-tetratic transition is replaced by an isotropic-nematic one. In this case, the nematic phase can exist even for the case of squares $(L / B=$ 1 ). The discontinuous transition observed at intermediate $L / B$ also becomes continuous for large enough $v$, but, curiously, the increase of $S$ with $\phi^{*}$ near the transition becomes more abrupt when $v$ increases.

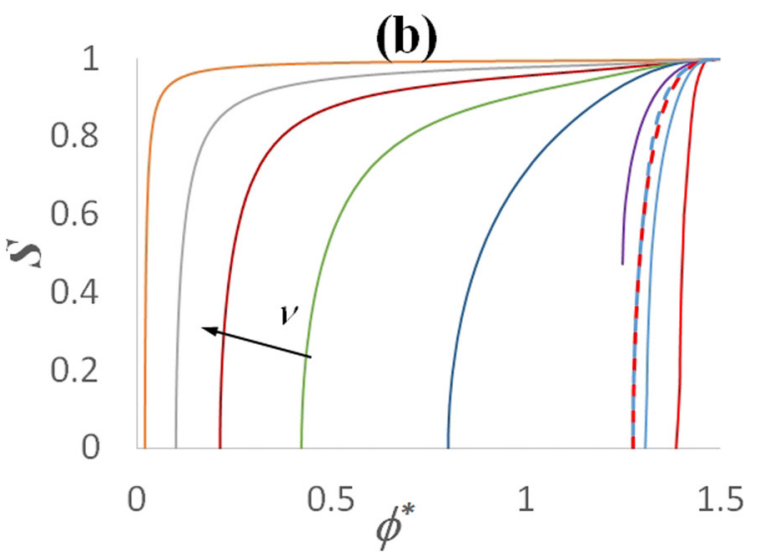

FIG. 2. Order parameter as a function of the scaled density, for $L / B=10000$ (a) and 1.5 (b), and the following values of the quadrupolar order parameter (increasing as indicated by the arrow): (a) $0,0.0625,0.175,0.45,1.25,3.75$, and 16 and (b) $0,0.5,1.2,2.3,4.65,10$, and 50 . 


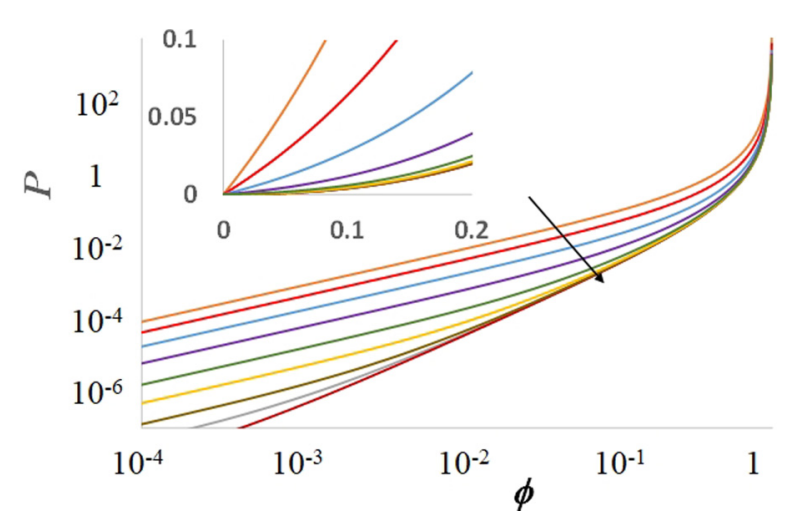

FIG. 4. Pressure of the isotropic phase as a function of area density, for a system with no interaction and the following values of $L / B$ (increasing in the direction of the arrow): 1, 2, 5, 15, 60, 200, 800,2500 , and 10000 . The inset shows the region of small pressure, in linear scale.

\section{B. Equation of state}

First, the dimensionless pressure of the isotropic phase in the whole composition range is considered. Although when the surface density is higher than $\phi_{\mathrm{IN}}$ the ordered phase represents the equilibrium state, this analysis provides a general insight and will allow one to distinguish order-dependent factors that affect the equation of state from order-independent ones.

The effect of $L / B$ on the equation of state for an athermal system is shown in Fig. 4. In a system with no interactions, two terms contribute to the pressure: the first and third terms of the right-hand side of Eq. (12). As $\rho$ is proportional to $L / B$, the third term becomes more important as $L / B$ increases and all the curves converge in a very large range of $\phi$ for large $L / B$. In this condition the pressure is quadratic with $\phi$, except for very large (where it diverges) or small density. As $\phi$ approaches zero, the first term is linear and dominates over the third one, and the system behaves as an ideal gas in a range of density that increases as $L / B$ decreases.

Figure 5 shows the effect of interactions on the pressure of the isotropic phase, for different values of $L / B$. The quadrupolar parameter $v$ has, obviously, no effect in this case where no nematic order is considered. For small enough density, the first term prevails and the pressure approaches the athermal behavior; it deviates from this limit at smaller values of $\phi$ as $\chi$ increases. Increasing interaction decreases the pressure (positive $\chi$ implies attraction between the particles). Note that the interaction term in the pressure, which is negative, is quadratic in $\phi$ and does not diverge for $\phi=1$ so it can become dominant only in some intermediate range of $\phi$. As it is very well known in fluid phase equilibria, for large enough $\chi$ the pressure develops a horizontal inflection point, which corresponds to the critical point (in Fig. 5 the curves
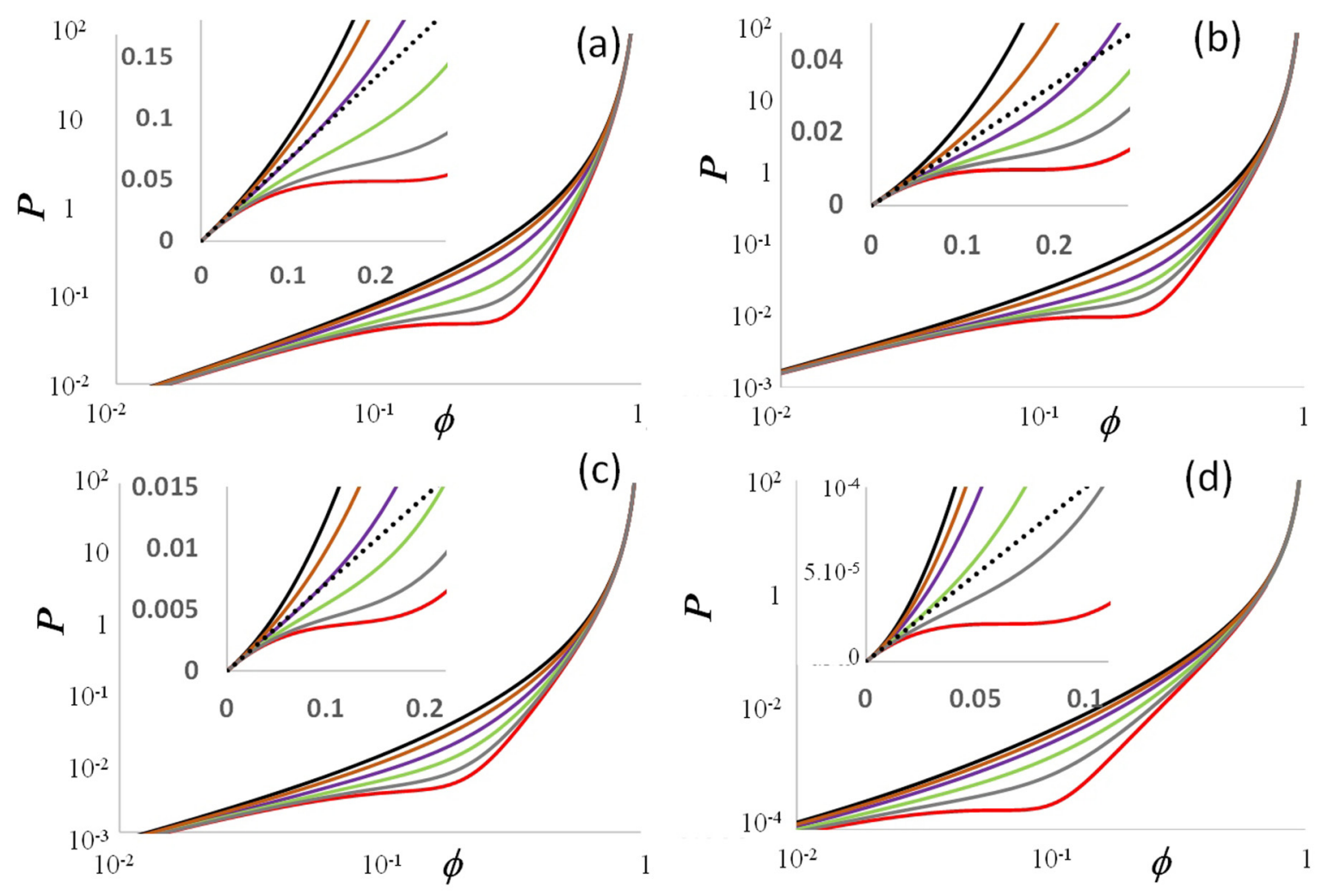

FIG. 5. Surface pressure of the isotropic phase as a function of area density. The insets show the behavior for small pressures, in linear scale, where ideal behavior is plotted as a dotted line. (a) $L / B=1.5 ; \chi=0,0.75,1.9,3.2,3.9$, and 4.3 . (b) $L / B=6 ; \chi=0,0.5,0.975,1.2$, 1.33, and 1.47. (c) $L / B=14 ; \chi=0,0.26,0.52,0.7,0.82$, and 0.91 . (d) $L / B=100 ; \chi=0,0.1,0.2,0.335,0.425$, and 0.485 . For a given surface density, increasing $\chi$ decreases the pressure. The last $\chi$, corresponding to red curves (in the online color version) with the horizontal inflection point, is the critical $\chi$ for each $L / B$. 


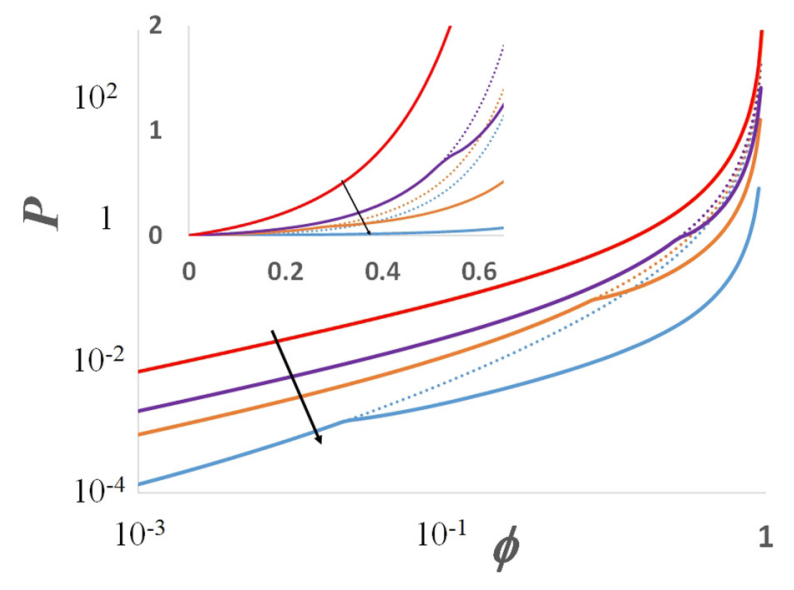

FIG. 6. Surface pressure a function of area density, for a system with no interaction and the following values of $L / B$ (increasing in the direction of the arrow): $1.5,6,14$, and 100 . The inset shows the region of small pressure, in linear scale. The dotted line represents the metastable isotropic phase in conditions where the order phase is the equilibrium one.

corresponding to this critical value, $\chi_{c}$, are plotted in red color). When $\chi$ is larger than this value, there is a range of $\phi$ where a single isotropic phase is unstable with respect to phase separation. A detailed study of phase separation is out of the scope of the present paper and it will be presented in a future publication.

As both the interaction and excluded volume contributions have opposite signs and a similar dependence on $\phi$ (at least in some range), they might cancel each other to a certain extent. This can be easily seen in Eq. (12), where, for the isotropic phase,

$$
P=\left(\frac{L}{B}\right)^{-1}\left[\frac{\phi}{(1-\phi)}+\left(\frac{1+2\left(\frac{L}{B}\right)^{-1}+\left(\frac{L}{B}\right)^{-2}}{\pi(1-\phi)^{2}}-\chi\right) \frac{L}{B} \phi^{2}\right] .
$$

In the insets of Fig. 5, the behavior of ideal gas [only the first term in Eq. (12) or Eq. (13)] is shown with dotted lines, and it observed that, indeed, the value of $\phi$ at which the system starts departing from ideality is maximum for a nonzero value of $\chi$.

Next, the nematic phase is considered in the equation of state. First, the effect of $L / B$ is considered for an athermal system; this is shown in Fig. 6. The pressure of the ordered phase is smaller than that in the isotropic phase, which is shown with dotted lines for $\phi>\phi_{\text {IN }}$ (in this range the isotropic phase is metastable). The pressure difference between both phases increases with $L / B$. This is because longer particles have a stronger tendency to form a nematic phase, with a larger decrease in the free energy. As a consequence of these two
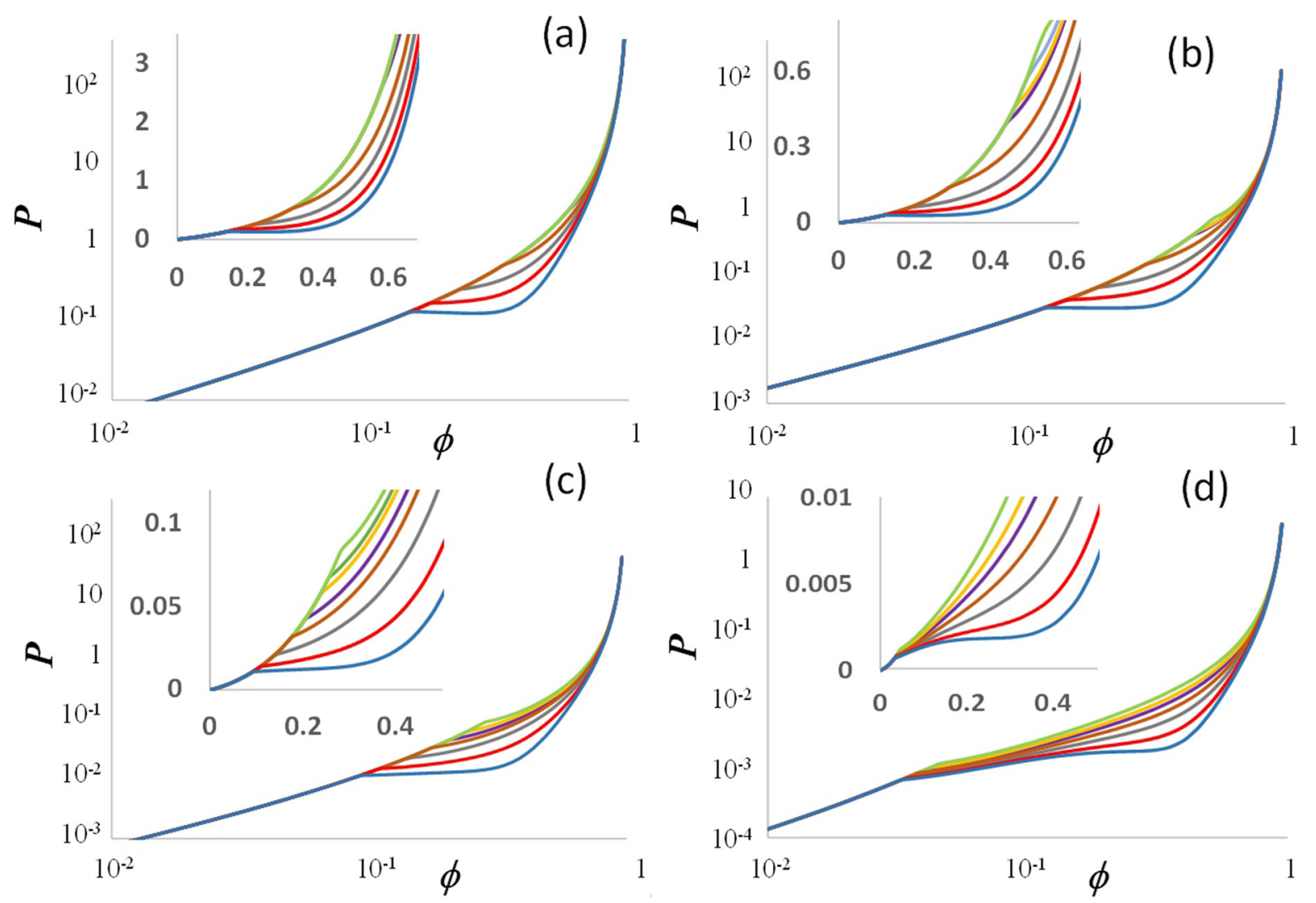

FIG. 7. Surface pressure as a function of area density for systems without isotropic interactions $(\chi=0)$. The insets show the behavior for small pressures, in linear scale. (a) $L / B=1.5 ; v=0,0.65,1.1,2,3,3.9$, and 4.65 (note that the first few values are difficult to distinguish due to the proximity of the different curves, and some are not shown in the inset). (b) $L / B=6 ; v=0,0.125,0.25,0.45,0.76,1$, and 1.22 . (c) $L / B=14 ; v=0,0.06,0.12,0.185,0.3,0.45$, and 0.575 . (d) $L / B=100 ; v=0,0.02,0.033,0.05,0.0675,0.083$, and 0.093 . Increasing $v$ decreases the pressure at constant surface density. 
contributions, the effect of $L / B$ on the pressure of the nematic phase is stronger than in the isotropic phase.

Figure 7 shows the effect of anisotropic interactions on the equation of state. For low $\phi$, the system is isotropic and pressure is obviously not affected; in the ordered phase the pressure decreases as $v$ increases. When $v$ is large enough, the nematic phase develops an instability, as observed by the formation of horizontal inflections, and for larger values of $v$ there is phase coexistence (which could be isotropic-nematic or nematic-nematic, to be analyzed in a future publication). As $L / B$ increases, smaller increases in $v$ produce a larger decrease in pressure (same as observed before for the degree of ordering).

Figure 8 shows the effect of isotropic interaction in the pressure of the nematic phase. Although, as mentioned before, $\chi$ does not affect the degree of ordering, the pressure in the nematic phase has contributions coming from both the nematic and the isotropic free energy terms as observed in Eq. (12). Again, increasing the interaction parameter decreases the pressure, and the development of an instability leading to isotropic-nematic or nematic-nematic phase coexistence is observed. As for the nematic interactions, for larger $L / B$, smaller values of $\chi$ produce a larger decrease on pressure. It is interesting to observe that, as $L / B$ increases, the instability in the nematic phase is developed not only for smaller values of $\chi$ in terms of its absolute value but also for smaller $\chi / \chi_{c}$ (the largest values of $\chi / \chi_{c}$ considered in Fig. 8 are 1 for $L / B=6$, 0.57 for $L / B=14$, and 0.2 for $L / B=100$ ). This means that the effect of $\chi / \chi_{c}$ on the shape of the $P$ vs $\phi$ curve in the nematic phase is dependent on $L / B$. Curiously, this is not the case for the isotropic phase, as observed in Fig. 5, where the shapes of the curves are very similar for different values of $L / B$.

\section{CONCLUSIONS}

A generalized van der Waals equation for a system of 2D particles with shape anisotropy, based in scaled particle theory and considering both isotropic and anisotropic quadrupolar Maier-Saupe interactions, was developed and solved in the case of rectangles, considering nematic and tetratic ordering. Phase transition points, degree of ordering, and equation of state behavior were analyzed as a function of the model parameters: aspect ratio $L / B$, and interaction parameters $\chi$ and $v$. Increasing $L / B$ and $v$ favors the formation of nematic ordering, decreasing the surface density for the onset of nematic ordering, $\phi_{\mathrm{IN}}$, and increasing the order parameter. For large $L / B$, the isotropic-nematic transition is continuous, and as this parameter approaches infinity the behavior approaches the "Onsager" case. In an intermediate range of $L / B$ and for small $\nu$, the nematic isotropic transition becomes discontinuous, and for small $L / B$ a continuous isotropic to tetratic transition is observed, followed by a (also continuous) tetratic to nematic transition as $\phi$ is increased.

The pressure of the nematic phase is smaller than that of the isotropic phase. Increasing $L / B$ decreases pressure; this effect is stronger in the nematic phase due to the fact that the nematic contribution to the free energy is proportional to $L / B$. The effect of both types of interactions, isotropic and anisotropic, is to decrease the pressure both in isotropic and in ordered
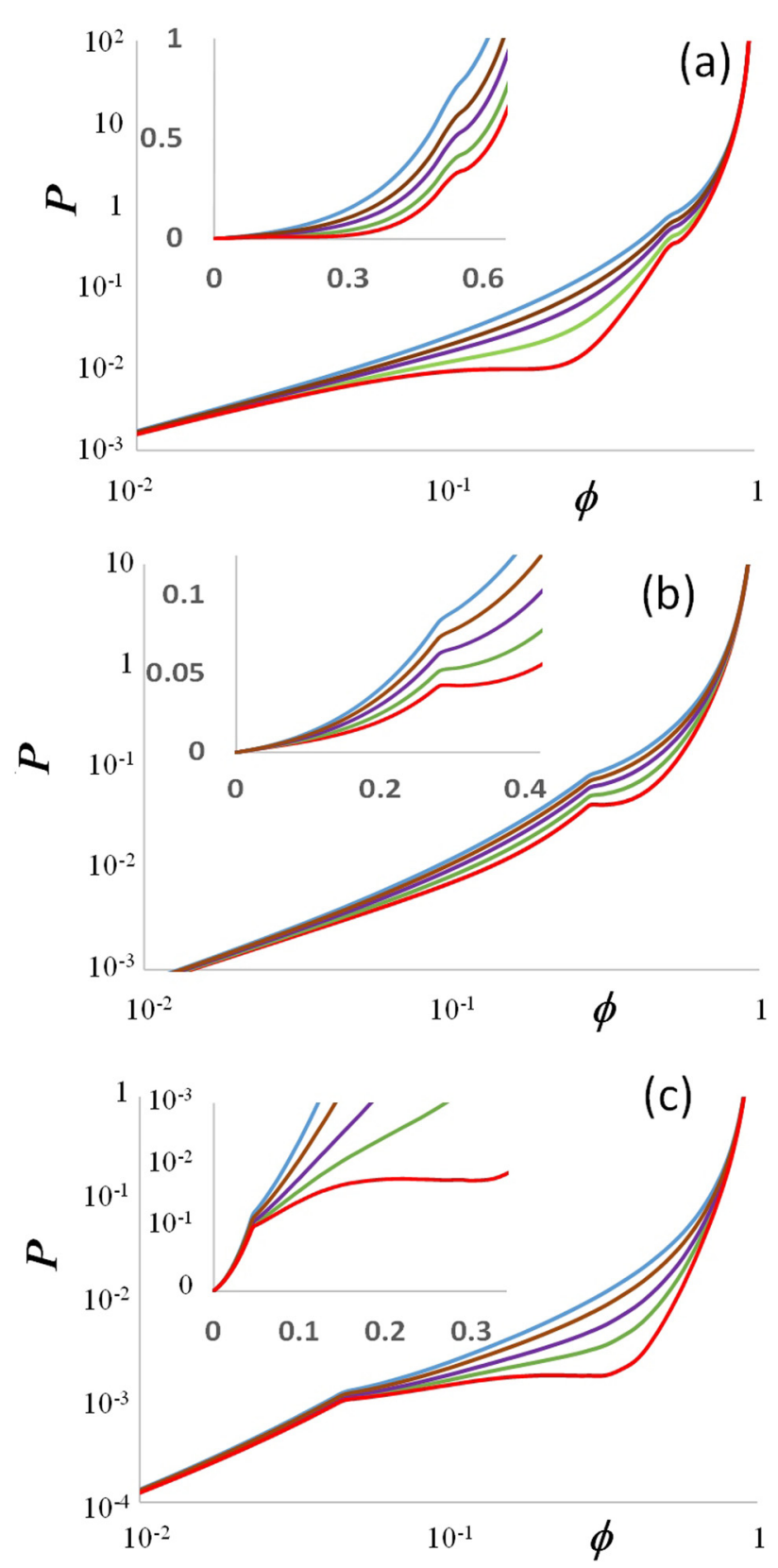

FIG. 8. Surface pressure as a function of area density for systems without nematic interactions $(v=0)$. The insets show the behavior for small pressures, in linear scale. (a) $L / B=6 ; \chi=0,0.5,0.825$, 1.2, and 1.47. (b) $L / B=14 ; \chi=0,0.13,0.26,0.4$, and 0.52 . (c) $L / B=100 ; \chi=0,0.03,0.06,0.08$, and 0.096. Increasing $v$ decreases the pressure at constant surface density. Results for $L / B=$ 1.5 are not shown as the curves are barely distinguishable from the isotropic case shown in Fig. 5.

phases. Regarding isotropic interactions, it was observed that, while in the isotropic phase, the qualitative shapes of $P$ vs $\phi$ curves are similar for similar values of $\chi / \chi_{c}$, which is not true for the nematic phase.

This relatively simple model allows a complete description of the equation of state behavior of the system, considering the particle geometry and two different types of interactions. In addition, it could be easily extended to account for other different particle shapes and other types of ordered phases. 


\section{ACKNOWLEDGMENTS}

This work was founded by the National Scientific and Technical Research Council, the National Agency for Promo- tion of Science and Technology, and the University of Mar del Plata. M.V.Z. acknowledges a scholarship from the Scientific Commission of Buenos Aires.
[1] P. F. F. Almeida and F. W. Wiegel, A simple theory of peptide interactions on a membrane surface: Excluded volume and entropic order, J. Theor. Biol. 238, 269 (2006).

[2] S. Jordens, K. Schwenke, I. Usov, E. Del Gado, and R. Mezzenga, Nematic field transfer in a two-dimensional protein fibril assembly, Soft Matter 12, 1830 (2016).

[3] S. Jordens, P. A. Ruhs, C. Sieber, L. Isa, P. Fischer, and R. Mezzenga, Bridging the gap between the nanostructural organization and macroscopic interfacial rheology of amyloid fibrils at liquid interfaces, Langmuir 30, 10090 (2014).

[4] S. Jordens, L. Isa, I. Usov, and R. Mezzenga, Non-equilibrium nature of two-dimensional isotropic and nematic coexistence in amyloid fibrils at liquid interfaces, Nat. Commun. 4, 1917 (2013).

[5] K. Slyusarenko, D. Constantin, and P. Davidson, A twodimensional nematic phase of magnetic nanorods, J. Chem. Phys. 140, 104904 (2014).

[6] Y. I. Yaman, E. Demir, R. Vetter, and A. Kocabas, Emergence of active nematics in chaining bacterial biofilms, Nat. Commun. 10, 2285 (2019).

[7] L. Onsager, The effects of shape on the interaction of colloidal particles, Ann. NY Acad. Sci. 51, 627 (1949).

[8] W. Maier and A. Saupe, Eine einfache molekular-statistische theorie der nematischen kristallinflüssigen phase. Teil II, Z. Naturforsch. Teil A 15, 287 (1960).

[9] W. Maier and A. Saupe, Eine einfache molekular-statistische theorie der nematischen kristallinflüssigen phase. Teil $1, \mathrm{Z}$. Naturforsch. Teil A 14, 882 (1959).

[10] J. D. Parsons, Nematic ordering in a system of rods, Phys. Rev. A 19, 1225 (1979).

[11] S.-D. Lee, A numerical investigation of nematic ordering based on a simple hard-rod model, J. Chem. Phys. 87, 4972 (1987).

[12] G. Jackson, M. Franco-Melgar, and A. J. Haslam, Advances in generalised van der Waals approaches for the isotropicnematic fluid phase equilibria of thermotropic liquid crystals-an algebraic equation of state for attractive anisotropic particles with the Onsager trial function, Mol. Phys. 107, 2329 (2009).

[13] H. H. Wensink and E. Trizac, Generalized Onsager theory for strongly anisometric patchy colloids, J. Chem. Phys. 140, 024901 (2014).

[14] H. H. Wensink and G. Jackson, Generalized van der Waals theory for the twist elastic modulus and helical pitch of cholesterics, J. Chem. Phys. 130, 234911 (2009).

[15] W. M. Gelbart and B. Barbo, A van der Waals picture of the isotropic-nematic liquid crystal phase transition, Acc. Chem. Res. 13, 290 (1980).

[16] H. Schlacken and M. J. Mogel, Orientational transitions of twodimensional hard rod fluids, Mol. Phys. 93, 777 (1998).
[17] J. A. Cuesta and D. Frenkel, Monte Carlo simulation of twodimensional hard ellipses, Phys. Rev A 42, 2126 (1990).

[18] O. Schönborn and R. C. Desai, Phase-ordering kinetics on curved surfaces, Physica A 239, 412 (1997).

[19] P. Biscari and E. Terentjev, Nematic membranes: Shape instabilities of closed achiral vesicles, Phys. Rev. E 73, 051706 (2006).

[20] Y. K. Murugesan and A. D. Rey, Modeling textural processes during self-assembly of plant-based chiral-nematic liquid crystals, Polymers (Basel) 2, 766 (2010).

[21] Y. K. Murugesan, D. Pasini, and A. D. Rey, Self-assembly mechanisms in plant cell wall components, J. Renew. Mater. 3, 56 (2015).

[22] G. Napoli and L. Vergori, Surface free energies for nematic shells, Phys. Rev. E 85, 061701 (2012).

[23] J. F. Nagle, Theory of the main lipid bilayer phase transition, Annu. Rev. Phys. Chem. 31, 157 (1980).

[24] H. S. Guidi and V. B. Henriques, Lattice solution model for order-disorder transitions in membranes and Langmuir monolayers, Phys. Rev. E 90, 052705 (2014).

[25] F. O. De Oliveira and M. N. Tamashiro, Phase transitions in phospholipid monolayers: Statistical model at the pair approximation, Phys. Rev. E 99, 012147 (2019).

[26] M. A. Bates and D. Frenkel, Phase behavior of twodimensional hard rod fluids, J. Chem. Phys. 112, 10034 (2000).

[27] D. Frenkel and R. Eppenga, Evidence for algebraic orientational order in a two-dimensional hard-core nematic, Phys. Rev. A 31, 1776 (1985).

[28] J. A. Cuesta, C. F. Tejero, and M. Baus, Isotropicnematic transition of hard ellipses, Phys. Rev. A 39, 6498 (1989).

[29] A. Chrzanowska, On the application of the Onsager DFT theory to two-dimensional system of hard needles, Acta Phys. Pol. B 36, 3163 (2005).

[30] H. H. Wensink and R. L. C. Vink, First-order phase transitions in two-dimensional off-lattice liquid crystals, J. Phys. Condens. Matter 19, 466109:1 (2007).

[31] R. L. C. Vink, Liquid crystals in two dimensions: First-order phase transitions and nonuniversal critical behavior, Phys. Rev. Lett. 98, 217801 (2007).

[32] J. Geng and J. V. Selinger, Theory and simulation of twodimensional nematic and tetratic phases, Phys. Rev. E 80, 011707 (2009).

[33] T. Heinemann, M. Antlanger, M. Mazars, S. H. L. Klapp, and G. Kahl, Equilibrium structures of anisometric, quadrupolar particles confined to a monolayer, J. Chem. Phys. 144, 074504 (2016). 